\title{
A Pesquisa de Sangue Oculto nas Fezes Associada a um Questionário de Sinais e Sintomas na Prevenção do Câncer Colo Retal
}

\author{
Faecal Occult Blood (FOB) Test Associated with a Questionaire of Signs and \\ Symptoms in Screening for Colorectal Cancer
}

\author{
FRANCISCO LUIS ALTENBURG ${ }^{1}$, MARIADE LOURDES PESSOLE BIONDO-SIMÕES $^{2}$, LUIS CARLOS VON BAHTEN $^{3}$ \\ 1. Mestrando do Programa de Pós-Graduação em Clínica Cirúrgica da PUCPR, Coordenador do Serviço de \\ Coloproctologia do Hospital Regional Hans Dieter Schmidt, Joinville, Santa Catarina; ${ }^{2}$ Professora Titular de \\ Metodologia da Pesquisa da PUCPR e Professora Adjunto IV do Departamento de Cirurgia da Universidade Federal \\ do Paraná. Membro da Sociedade Brasileira de Colo-Proctologia e Titular do Colégio Brasileiro de Cirurgiões \\ $(C B C) ;{ }^{3 .}$ Professor Coordenador e Orientador do Programa de Pós-Graduação em Clínica Cirúrgica da PUCPR, \\ Membro Titular do CBC.
}

\begin{abstract}
ALTEMBURG FL; BIONDO-SIMÕES MLP; VON BAHTEN LC. A Pesquisa de Sangue Oculto nas Fezes Associada a um Questionário de Sinais e Sintomas na Prevenção do Câncer Colo Retal. Rev bras Coloproct, 2009;29(1): 057-064.

RESUMO: OBJETIVO: Determinar a eficácia de um programa de prevenção de Câncer Colo Retal (CCR) através do método da pesquisa de sangue oculto das fezes (PSO), e um questionário clínico (QSA). Material e Método: Foi realizada uma triagem através do PSO e do QSA em indivíduos acima de 50 anos, oriundos de uma campanha de prevenção de CCR em Joinville, SC. 387 indivíduos aderiram à campanha e 93 foram excluídos.. Foram definidos 3 grupos: 1- PSO +, com 20 sujeitos, QSA +, com 38 sujeitos e PSO e QSA negativos com 21 sujeitos. Foi definida como colonoscopia positiva aquela que detectou adenomas. Resultados: $O$ grupo 1 apresentou $22 \%$ de sensibilidade, $74 \%$ de especificidade, likelihood ratio positivo (LR) de 0,9 , acurácia de 56, 9, valor preditivo positivo (VPP) de 31,6 e negativo (VPN) de 64. O grupo 2 apresentou 71,4\% de sensibilidade, 45,6 de especificidade, LR de 1,3, acurácia de 52,6, VPP de 32,6 e VPN de 81,3. O método apresentou 95,2\% de sensibilidade, 63,2 de especificidade, LR de 2, 6, acurácia de 71, 8, VPP de 48.8 e VPN de 97.3. Conclusão: O método da PSO e QSA são complementares e efetivos como triagem em programas de prevenção de CCR.
\end{abstract}

Descritores: Neoplasias do Colon, Diagnóstico Colonoscopia, Anamnese, Promoção da Saúde.

\section{INTRODUÇÃO}

O câncer colo-retal (CCR) representa uma das mais significantes e prevalentes causas de morte por câncer no dias atuais. Nos Estados Unidos e no Reino Unido é o terceiro tipo de câncer mais diagnosticado e a segunda causa de morte por câncer ${ }^{1}$.

No Brasil, para o ano de 2008, o Instituto Nacional do Câncer (INCA) projetou 12.490 novos casos em homens e 14.500 novos casos em mulheres, ocu- pando a quarta e a terceira colocação respectivamen$\mathrm{te}^{2}$.

Programas de prevenção têm-se mostrado efetivos na diminuição de mortalidade e incidência por câncer colo-retal, sendo recomendados por várias instituições proeminentes por todo o mundo ${ }^{3}$. Apesar disto, os índices de participação permanecem baixos, influenciando o custo efetividade dos $\operatorname{programas}^{1,3}$.

A colonoscopia é o melhor exame preventivo, pois além de diagnosticar o CCR permite identificar e

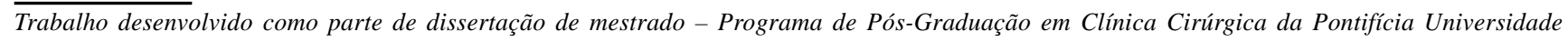
Católica do Paraná (PUCPR). 
A Pesquisa de Sangue Oculto nas Fezes Associada a um Ouestionário de Sinais e Sintomas na Prevenção do Câncer Colo Retal Francisco Luis Altemburg e Cols. tratar pólipos, lesões precursoras da doença, sendo responsável pelo declínio da incidência do CCR, pós 1986, nos EUA 4 .

São necessários, porém métodos de triagem para a realização de colonoscopias, pois mesmo em países desenvolvidos não seria possível à realização de colonoscopias em todos os indivíduos acima de 50 anos, porém o custo benefício da diminuição de mortalidade pela detecção precoce das lesões precursoras (pólipos), ou os crescentes achados de CCR em fase inicial têm estimulado a realização destas triagens ${ }^{3}$.

A maioria dos programas de prevenção de CCR é baseada em triagens realizadas pela pesquisa de sangue oculto nas fezes. Recente revisão sistemática da Cochrane demonstrou que o rastreamento feito com a pesquisa de sangue oculto nas fezes reduziu o risco relativo para o CCR em $16 \%$. A redução do risco relativo de morte por CCR foi de $15 \%$. Já a redução do $\mathrm{RR}$ de morte em indivíduos de risco chegou a $25 \%{ }^{1}$.

Recente metanálise reportou que a PSO não teve impacto na mortalidade, nem foi estatisticamente significante em relação às mortes por outras causas na população triada. A maioria dos estudos revelou alta porcentagem de falsos positivos. Para eles o impacto da PSO na mortalidade é incerto e sua eficácia no rastreamento do CCR necessita de reavaliação ${ }^{5}$.

Outra metanálise de revisão de mortalidade em grupos controlados de rastreamento de CCR, realizado com PSO mostrou redução de mortalidade de $14 \%$ em 10 anos. Entretanto, o acompanhamento a longo prazo não demonstrou proteção ${ }^{6}$.

Quando se analisa o custo efetividade de vários programas de prevenção verifica-se que a maioria deles inclui indivíduos com mais de cinqüienta anos, que apresentam risco aumentado para o CCR. A sigmoidoscopia flexível e a colonoscopia parecem ter o melhor custo efetividade. Este fato é devido ao conceito assumido de que praticamente todos os CCR, provêm de adenomas, que podem ser detectáveis ${ }^{7}$.

O segmento de indivíduos com potencial risco para CCR, durante 11 anos, rastreados pelo PSO, permitiu verificar que os programas de prevenção baseados neste exame permitiram diminuir a incidência da doença. Nos indivíduos que se recusaram a realizar o exame a incidência de CCR foi maior. É bem provável que entre os rastreados a incidência tenha sido maior pela realização de maior número de colonoscopias complementares. Neste estudo os autores utilizaram um questionário de rastreamento ${ }^{8}$.
No Reino Unido, questionários baseados em sintomas (WNS) têm sido utilizados para abreviar o tempo de espera para consultas com especialistas em cirurgia colo-retal (fast track referral)l. Vários estudos foram feitos e verificaram a efetividade deste sistema de referência e recomendaram a continuação do programa. Estudos que relatam a importância de se coletar dados em um programa de rastreamento de CCR como: sintomas, história familiar, pessoal e fatores de risco, comprovam que esta coleta de dados aumenta a adesão a programas de prevenção com a pesquisa de sangue oculto nas fezes ${ }^{9,10}$.

A ideia de coletar informações de sintomas e antecedentes não é nova. Keddie e Hargreaves, em 1968, já reconheceram que não havia relação entre o início de sintomas e o estádio do CCR, e que a presença de sintomas sistêmicos eram comuns ${ }^{11}$. Outros estudos avaliam o tempo de evolução dos sintomas até a descoberta do $\mathrm{CCR}^{12}$, a presença de sintomas precoces nos indivíduos com adenomas e CCR, e a importância da história familiar nos programas de prevenção ${ }^{13,14}$.

A hipótese de que a descoberta de lesões neoplásicas do cólon e reto poderia ser incrementada com um questionário abordando sinais, sintomas e antecedentes, entre nós, foi levantada a partir de um estudo piloto no qual se analisou os resultados de colonoscopias em indivíduos com PSO positivo e negativo. Aquele estudo revelou que a maioria dos indivíduos triados e com colonoscopia positiva já eram sintomáticos ${ }^{15}$.

\section{OBJETIVO}

O objetivo deste trabalho foi verificar a eficácia e acurácia da PSO associada a um questionário clínico (QSA), com enfoque em sintomas de alarme do CCR, como meio de triagem para o diagnóstico de neoplasias do cólon e reto.

\section{MÉTODOS}

O projeto que deu origem a este estudo obedeceu a Resolução do Conselho Nacional de Saúde 196/ 96 e foi avaliado e aprovado pela Comissão de Ética do Hospital Regional de Joinville, com o número 08009.

A população estudada foi proveniente de uma triagem realizada em uma campanha de prevenção do câncer do cólon e reto, em que foram atingidos por 
A Pesquisa de Sangue Oculto nas Fezes Associada a um Ouestionário de Sinais e Sintomas na Prevenção do Câncer Colo Retal Francisco Luis Altemburg e Cols. correspondência direta, fôlderes e por meios de comunicação, aproximadamente 10.000 pessoas acima dos 50 anos de idade, de ambos os sexos, usuárias de um plano de saúde local, que voluntariamente aderiram ao programa de prevenção.

A pesquisa clínica foi realizada nas instalações de um centro hospitalar (CHU), de um plano de saúde local. No período de 21 de novembro a 10 de dezembro de 2007 foi realizada a campanha de prevenção do CCR durante a qual foram preenchidos os questionários (QSA) e realizados os exames de PSO. Foram excluídos do estudo os sujeitos que já tinham realizado colonoscopia anteriormente.

O método da PSO utilizado foi o imunocromatográfico para todas as amostras examinadas.

O detalhamento do QSA está no anexo 1. Este foi considerado positivo para dois critérios:

1- quando da existência de antecedentes familiares, isto é, a presença de pelo menos um familiar, de primeiro grau, com CCR ou adenomas;

2- quando da existência de sintomas:

2a- específicos - Sangue misturado às fezes e/ ou alteração de hábito intestinal por mais de três meses para mais frequente, fezes finas e, ou perda de fezes (estes dois sintomas isolados determinavam um QSA positivo),

2b- não específicos - dor abdominal em hipogástrio por mais de três meses, anemia sem causa definida, astenia, dor retal e sensação de evacuação incompleta.

Nos casos de sintomas não específicos o QSA foi considerado positivo quando havia a associação de pelo menos dois sintomas.

A partir da análise do QSA e dos resultados da PSO foram definidos três grupos:

Grupo 1- Indivíduos com PSO positiva;

Grupo 2- Indivíduos com QSA positivo;

Grupos 3- Voluntários com PSO negativa e QSA negativo.

Foram realizadas colonoscopias nos pacientes dos três grupos que foram feitas nas instalações do CHU, sob sedação profunda, com acompanhamento de um anestesiologista. Para a sedação foi utilizado Propofol ( $1 \mu \mathrm{g} / \mathrm{Kg})$, fentanil (1 $\mu \mathrm{g} / \mathrm{Kg})$ e Midazolan (1 a $2 \mathrm{MG} / \mathrm{kg}$ ). Os endoscópios utilizados foram da marca Olympus ${ }^{\circledR}$
. Foi definida como colonoscopia positiva aquelas que detectaram lesões neoplásicas do cólon e/ ou reto. Este exame foi utilizado como controle do PSO e do QSA.

Os dados foram colocados no programa de banco de dados Excel e analisados pelo programa de análise estatística 1.8 para Windows ${ }^{\circledR}$, reconhecendose a sensibilidade, a especificidade, os valores preditivos positivo e negativo, o likelihood ratio e a acurácia dos exames nos grupos de estudo.

\section{RESULTADOS}

Aderiram ao estudo 387 indivíduos. Destes 93 foram retirados do estudo por apresentarem critérios de exclusão. Restaram para análise 294 indivíduos, 138 $(46,93 \%)$ do sexo masculino e 156 do sexo feminino $(53,03 \%)$. A média de idade foi $59,02 \pm 14,42$ anos. A idade mínima foi de 50 anos e a máxima foi de 90 anos. A idade, o peso e o índice de massa corpórea (IMC) em cada um dos grupos foram semelhantes, o que deu caráter homogêneo à amostra estudada.

Para uma população estimada de 10.000 nesta faixa etária, o grau de adesão ao programa foi de apenas $3,87 \%$.

Após a análise do questionário estabeleceramse os participantes de cada grupo. No grupo 1 ficaram 20 indivíduos, no grupo 2 restaram 47 e no grupo 3, 21 participantes.

Foram realizadas 78 colonoscopias e todas alcançaram o íleo terminal. A distribuição dos sujeitos da pesquisa, e os resultados das colonoscopias estão descritos no organograma da figura 1 .

Os resultados estão demonstrados na tabela 1 .

Em relação ao grupo 3(QSA negativo e PSO negativo), foi diagnosticado um (1) único pólipo adenomatoso. Neste grupo a sensibilidade foi de $4,8 \%$ e a especificidade de $64,9 \%$, valor preditivo positivo de $4,8 \%$ e negativo de $64,9 \%$, com acurácia de $48,7 \%$. Likelihood ratio para teste positivo de 0,1 e negativo de 1,5 .

\section{DISCUSSÃO}

$\mathrm{Na}$ grande maioria dos casos de CCR os sintomas surgem quando a doença está avançada. Estes pacientes exigem maiores custos de tratamento e estão acompanhados de altas taxas de morbidade e mortalidade. 
A Pesquisa de Sangue Oculto nas Fezes Associada a um Questionário de Sinais e Sintomas na Prevenção do Câncer Colo Retal Francisco Luis Altemburg e Cols.
Vol. 29 $\mathbf{N}^{\mathbf{0}} 1$

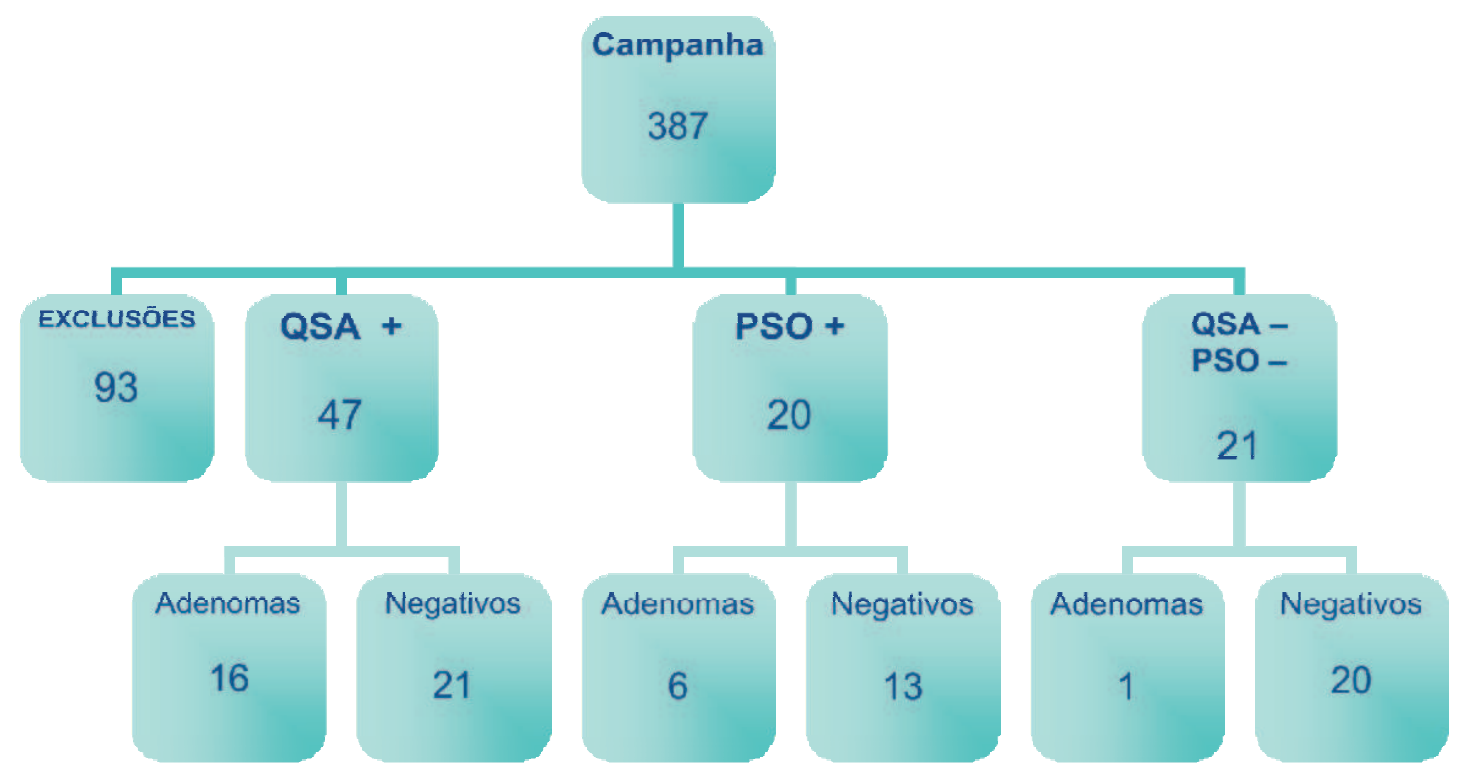

Figura 1 - Organograma demonstrativo da amostra e dos grupos de estudo.

O câncer colo-retal (CCR) representa uma das mais significantes e prevalentes causas de morte por câncer no dias atuais. Nos Estados Unidos e no Reino Unido é o terceiro tipo de câncer mais diagnosticado, e a segunda causa de morte por câncer ${ }^{17}$. No Brasil, para o ano de 2008, o Instituto Nacional do Câncer (INCA) projetou 12.490 novos casos em homens e 14.500 novos casos em mulheres, ocupando a quarta e a terceira colocação respectivamente ${ }^{18}$.

Considerando a crescente incidência desta doença, seu diagnóstico precoce e das doenças predisponentes se torna importante. Programas de detecção são caros e limitantes para o sistema de saúde do Brasil.

O rastreamento do CCR tem se mostrado eficiente tanto na detecção precoce dos tumores, como na prevenção destes tumores pelo tratamento das lesões com potencial para malignização, como os pólipos adenomatosos ${ }^{16}$.
A pesquisa de sangue oculto nas fezes tem sido usada para rastreamento e acompanhamento de pacientes sem fatores de risco para câncer colo-retal ${ }^{1,6,8,19-}$ ${ }^{23}$. Os fatos demonstram que o exame anual de pesquisa de sangue oculto nas fezes pode diminuir em $16 \%$ a mortalidade por $\mathrm{CCR}^{22}$. Em um estudo publicado em 1993, os autores compararam o rastreamento anual com o bianual em uma população de 46.551 indivíduos com idade entre 50 e 80 anos, durante 13 anos. Relataram que a mortalidade por CCR nos acompanhados anualmente foi de 5,88: 1000 indivíduos, enquanto que nos acompanhados bianualmente foi de 8,33: 1000 indivíduos. Observaram que o rastreamento anual levou a diminuição de $33 \%$ da taxa de mortalidade ${ }^{24}$.

Um estudo publicado por Goodyear et al. no qual rastrearam indivíduos com idade entre 50 e 69 anos utilizando PSO, demonstrou $23 \%$ de redução na taxa de mortalidade por CCR. O dado importante deste estudo foi o diagnóstico de $49 \%$ dos pacientes sem

Tabela 1 - Demonstração da sensibilidade, da especificidade, da likelihood ratio(LR), da acurácia e dos valores preditivos positivo( $V P+)$ e negativo( $V P$-).

\begin{tabular}{lcccccc}
\hline & Sensibilidade \% & Especificidade \% & L.R & Acurácia \% & VP+ \% & VP- \% \\
\hline PSO + & 22 & 74 & 0,9 & 56,9 & 31,6 & 64,4 \\
QSA + & 71,4 & 45,6 & 1,3 & 52,6 & 32,6 & 81,3 \\
PSO + e QSA + & 95,2 & 63,2 & 2,6 & 71,8 & 48,8 & 97,3 \\
\hline
\end{tabular}


Rev bras Coloproct Janeiro/Março, 2009
A Pesquisa de Sangue Oculto nas Fezes Associada a um Questionário de Sinais e Sintomas na Prevenção do Câncer Colo Retal Francisco Luis Altemburg e Cols.
Vol. 29 sintomatologia o que permitiu o diagnóstico de tumores em fases iniciais ${ }^{25}$.

Ao se analisar a resposta ao programa de prevenção realizado, verificou-se um baixo índice de adesão $(3,87 \%)$, confirmando a tendência de programas com adesão voluntária. Se considerarmos o alto custo da campanha pode-se pensar que o resultado não foi o esperado. Isto serve para que se tenha consciência de que se precisa trabalhar fortemente na mudança de conceito da população, precisa-se criar a cultura da prevenção. Segundo Trevena et al., em um estudo desenvolvido na Austrália com indivíduos com idade entre 50 e 74 anos, o nível cultural-educacional tem importante papel determinante na adesão dos indivíduos aos programas de rastreamento. Demonstraram que a adesão daqueles que tinham melhor formação chegou a $50 \%$, e a $79,4 \%$ entre aqueles com formação universitária $^{26}$.

A PSO (baseada no guáiaco e os testes imunológicos), a sigmoidoscopia flexível e a colonoscopia são recomendadas para o rastreamento. Segundo Sung et al. a PSO é a primeira escolha para os países com recursos limitados ${ }^{27}$.

Estudo recente revelou taxa de positividade da PSO de 2,4\%. A identificação de neoplasia nestes indivíduos atingiu 22,92\%. O emprego de testes imunoquímicos elevou a taxa de positividade para 5,5\%, e a identificação de neoplasias nestes indivíduos alcançou $19,93 \%{ }^{28}$. Um estudo desenvolvido na República Checa envolvendo 535.248 indivíduos demonstrou positividade da PSO de $6,4 \%{ }^{29}$. Outro estudo realizado na Itália envolvendo 827.473 indivíduos revelou 5,8\% de exames psitivos ${ }^{30}$. No estudo ora apresentado, apesar de se ter baixo percentual de aderência, a taxa de positividade seguiu a tendência descrita, com taxa de $6,8 \%$ de exames positivos.

A porcentagem de indivíduos com QSA positivo foi de 12,95 , sendo que destes $34 \%$ dos pacientes eram portadores de adenomas. Segundo Selvachandran et al. o questionário e o score numérico podem ter lugar na prática geral identificando indivíduos com substancial risco para o $\mathrm{CCR}^{9}$.

No presente levantamento verificou-se que o método de rastreamento pela PSO apresentou baixa sensibilidade (22\%), com razoável especificidade (74\%). O QSA, porém, apresentou sensibilidade de 71,4\% com especificidade de $45.6 \%$. Entretanto a combinação dos dois métodos levou para 95,2\% a sensibilidade, e para $63.2 \%$ a especificidade, sendo que a acurácia melhorou em média para os métodos isolados de 52 para $71.8 \%$,sendo que o likelihood ratio positivo foi de 2.6 , o que representa que quando a colonoscopia é positiva há $2.6 \mathrm{x}$ mais chance do método ser também positivo. Quando se associam os dois modos de rastreamento o valor preditivo positivo foi de $48,8 \%$, entretanto o valor preditivo negativo chegou a $97,3 \%$. Desta forma pacientes com PSO negativa e questionários negativos teriam $97,3 \%$ de chance de realmente não serem portadores de lesões neoplásicas. Observa-se que os métodos da PSO e do QSA são complementares melhorando todos os índices de eficácia analisados. Considerando as dificuldades econômicas dos países em desenvolvimento, a associação dos dois métodos poderia ser um bom método de rastreamento do CCR. Entretanto, estudos, no nosso meio, devem ser ampliados para poder oferecer maior segurança de efetividade nos programas de detecção precoce do CCR, além de ter caráter educativo em relação à divulgação dos sintomas e sinais, melhorando a taxa de adesão a estes programas.

\section{CONCLUSÃO}

A utilização da pesquisa de sangue oculto nas fezes combinada com o questionário de sinais e sintomas parece efetiva como método de rastreamento do câncer colo-retal em programas de prevenção além de ter caráter educativo em relação à divulgação dos sinais, sintomas e antecedentes mais frequentes.

\footnotetext{
ABSTRACT: The purpose of this study is to verify the efficacy of a strategy to screen colorectal cancer(CCR) and adenomas using the faecal occult blood test(PSO) and a questionnaire (QSA)based in signs and symptoms of CCR. Methods: A program of prevention was done, and the patients answered the QSA and did the PSO type imunocromatograph. Results: 387 people adhered do the program, 93 were excluded. Three groups were formed: 1: 20 persons, with PSO positive, 2:38 persons with QSA positive, and 3, with 21 persons with PSO and QSA negatives. Group 1 presented a sensibility of $22 \%$, specificity of $74 \%$, Likelihood ratio + of 0,9, accuracy of 56,9, Positive predictive value(VPP) of 31,6 and negative(VPN) of 64. Group 2 presented sensibility of 71,4\%, 45,6\% of specificity, Likelihood ratio + 1.3, accuracy of 52,6, VPP de 32,6 e VPN de 81,3. The QSA plus PSO method, presented a sensibility of 95,2\%, 63,2 of specificity, accuracy of 71,8,Likelihood ratio of 2,6, VPP de 48,8 e VPN 81,3. In conclusion the method of PSO and QSA are complementary and seem effective in screening for CCR in preventive programs.
} 
A Pesquisa de Sangue Oculto nas Fezes Associada a um Questionário de Sinais e Sintomas na Prevenção do Câncer Colo Retal Francisco Luis Altemburg e Cols.
Vol. 29

\section{REFERÊNCIAS}

1. Hewitson P, Glasziou P, Watson E, Towler B, Irwig L. Cochrane systematic review of colorectal cancer screening using the fecal occult blood test (hemoccult): an update. Am J Gastroenterol. 2008; 103(6):1541-9.

2. Instituto Nacional do Câncer (INCA). Estimativas 2008 Incidência de câncer no Braisil. Disponível em: http:// www.inca.gov.br/estimativas $/ 2008 /$ mapa.asp?ID=6. Acesso em: 7 Apr 2008.

3. Rothenberger DA, Dalberg DL, Leininger A. Minnesota Colorectal Cancer Initiative: successful development and implementation of a community-based colorectal cancer registry. Dis Colon Rectum. 2004; 47(10):1571-7.

4. Nelson RL, Persky V, Turyk M. Determination of factors responsible for the declining incidence of colorectal cancer. Dis Colon Rectum. 1999; 42(6):741-52.

5. Moayyedi P, Achkar E. Does fecal occult blood testing really reduce mortality? A reanalysis of systematic review data. Am J Gastroenterol. 2006; 101(2):380-4.

6. Heresbach D, Manfredi S, D'halluin PN, Bretagne JF, Branger B. Review in depth and meta-analysis of controlled trials on colorectal cancer screening by faecal occult blood test. Eur J Gastroenterol Hepatol. 2006; 18(4):427-33.

7. Pignone M, Rich M, Teutsch SM, Berg AO, Lohr KN. Screening for colorectal cancer in adults at average risk: a summary of the evidence for the U.S. Preventive Services Task Force. Ann Intern Med. 2002; 137(2):132-41.

8. Niv Y, Lev-El M, Fraser G, Abuksis G, Tamir A. Protective effect of faecal occult blood test screening for colorectal cancer: worse prognosis for screening refusers. Gut. 2002; 50(1):33-7.

9. Selvachandran SN, Hodder RJ, Ballal MS, Jones P, Cade D. Prediction of colorectal cancer by a patient consultation questionnaire and scoring system: a prospective study. Lancet. 2002; 360(9329):278-83.

10. Smith D, Ballal M, Hodder R, Soin G, Selvachandran SN, Cade D. Symptomatic presentation of early colorectal cancer. Ann R Coll Surg Engl. 2006; 88(2):185-90.

11. Keddie N, Hargreaves A. Symptoms of carcinoma of the colon and rectum. Lancet. 1968; 2(7571):749-50.

12. Stubbs RS, Long MG. Symptom duration and pathologic staging of colorectal cancer. Eur J Surg Oncol. 1986; 12(2):127-30.

13. Johns LE, Houlston RS. A systematic review and meta-analysis of familial colorectal cancer risk. Am J Gastroenterol. 2001; 96(10):2992-3003.

14. Church JM. A scoring system for the strength of a family history of colorectal cancer. Dis Colon Rectum. 2005; 48(5):889-96.

15. Altenburg FL, Biondo-Simões MLP, Santiago A. Pesquisa de sangue oculto nas fezes e correlação com alterações nas colonoscopias. Rev bras colo-proctol. 2007; 27(3): 304-9.

16. Levin B, Lieberman DA, McFarland B, Andrews KS, Brooks D, Bond J, et al. American Cancer Society Colorectal Cancer
Advisory Group; US Multi-Society Task Force; American College of Radiology Colon Cancer Committee. Screening and surveillance for the early detection of colorectal cancer and adenomatous polyps, 2008: a joint guideline from the American Cancer Society, the US Multi-Society Task Force on Colorectal Cancer, and the American College of Radiology. Gastroenterology. 2008; 134(5):1570-95.

17. Fang CB. Rastreamento do câncer colorretal. Rev Assoc Med Bras. 2002; 48(4):286-6.

18. www.inca.gov.br. Câncer colo-retal. Acesso em 24 ago 2008.

19. American Cancer Society. Cancer facts and figures, 1999. Atlanta: American Cancer Society, 1999.

20. Screening for colorectal cancer using the faecal occult blood test, Hemoccult. Cochrane Database Syst Rev. 2007 Jan 24;(1):CD001216.

21. Woo HY, Mok RS, Park YN, Park DI, Sung IK, Sohn CI, Park $H$. A prospective study of a new immunochemical fecal occult test in Korean patients referred for colonoscopy. Clin Biochem. 2005; 38(4):395-9.

22. Anderson WF; Guyton KZ; Hiatt RA; Vernon SW; Levin B; Hawk E. Colorectal cancer screening for persons at average risk. J Natl Cancer Inst. 2002;94(15):1126-33.

23. Ward N.,Wright J,Marsh S,Norton J. Fast track referrals for colorectal cancer: the impact of the faecal occult blood test screening program.Colorectal Disease.2007;9(3):258-61.

24. Mandel JS, Bond JH, Church TR, Snover DC, Bradley GM, Schuman LM, Ederer F. Reducing mortality from colorectal cancer by screening for fecal occult blood. Minnesota Colon Cancer Control Study. N Engl J Med. 1993; 328(19):1365-71.

25. Goodyear SJ, Stallard N, Gaunt A, Parker R, Williams N, Wong L. Local impact of the English arm of the UK Bowel Cancer Screening Pilot study. Br J Surg. 2008; 95(9):1172-9.

26. Trevena LJ, Irwig L, Barratt A. Randomized trial of a selfadministered decision aid for colorectal cancer screening. J Med Screen. 2008; 15(2):76-82.

27. Sung JJ, Lau JY, Young GP, Sano Y, Chiu HM, Byeon JS et al. Asia Pacific consensus recommendations for colorectal cancer screening. Gut. 2008; 57(8):1166-76.

28. van Rossum LG, van Rijn AF, Laheij RJ, van Oijen MG, Fockens $\mathrm{P}$, van Krieken $\mathrm{HH}$, et al. Random comparison of guaiac and immunochemical fecal occult blood tests for colorectal cancer in a screening population. Gastroenterology. 2008; 135(1):82-90.

29. Zavoral M. Colorectal cancer screening in the Czech Republic. Z Gastroenterol. 2008; 46 Suppl 1:S29-30.

30. Zorzi M, Barca A, Falcini F, Grazzini G, Pizzuti R, Ravaioli A et al. Screening for colorectal cancer in Italy: 2005 survey. Epidemiol Prev. 2007; 31(2-3 Suppl 2):49-60.

\section{Endereço para correspondência:}

FRANCISCO LUIS ALTENBURG

Rua 15 de novembro 2119, casa 14, Joinville, Santa Catarina CEP 89201-620

Tel.: (047) 3422-9363 / (047) 9964-5837

E mai: Progastro@terra.com.br 
Anexo 1 - Questionário de Sinais, Sintomas e Antecedentes.

Preencha os campos abaixo e assinale x nos parênteses (frente e verso)

Nome

Idade

Sexo

Endereço

Telefone para contato

Altura:

Peso (aproximado)

1: Já notou sangue nas fezes? A-( ) sim B-( ) não

Caso tenha respondido sim, assinale nos espaços abaixo. Se repondeu não, deixe os campos em branco.

C-sangue vivo

( )

D-sangue escuro

( )

E-sangue no meio das fezes

( )

F-sangue no papel separado das fezes

( )

G-sangue misturado e separado

( )

H-não sei identificar o tipo do sangramento

( )

Quão freqüente é o sangramento?(assinale x nos parênteses)

I- todos os dias ( )

$\mathrm{K}$ - duas vezes por semana ( )

J- sangrou uma vez ou raramente ( )

O sangramento começou:

$\mathrm{N}$ - há uma semana ou menos

O-há um mês

P- há menos de 3 meses

Q- há um ano

O sangramento é:

$\mathrm{R}$ - pequena quantidade

$\mathrm{S}$ - grande quantidade
J- uma vez por semana ( )

1- uma vez por mês ( )

Você tem sangramento, sente alguma coisa no ânus?Coceira, dor, ou aumento de volume (inchaço ou tumor ação)

T- $\operatorname{Sim}()$ U- Não ( )

2- Como é o seu habito intestinal normalmente?(quantas vezes evacua?).
A- Diário ( )
B- Mais de uma vez ao dia ( )
C- Duas vezes por semana ( )
D- Três vezes por semana ( )
E- Menos de uma vez por semana ( )

Normalmente seu intestino é:

F- Preso (constipado, fezes duras)

G- Diarreia

H- Alterna diarréia e preso

Seu hábito intestinal mudou nos últimos tempos?

I- Sim ( )

J- Não ( ) 
Se mudou como mudou? RESPONDA APENAS SE MUDOU

$\mathrm{K}$ - Mais frequente (evacuo mais vezes) (

L- Para diarreia ( )

M- Ficou mais preso ( )

$\mathrm{N}$ - Estou perdendo fezes ( )

O- Tenho urgência ( )

P- Agora tenho fezes finas ( )

Há quanto tempo mudou?

Q- Mais de 3 meses ( )

R- Menos de 3 meses ( )

3- Você vê muco (geleia) ou pus nas fezes?
A- $\operatorname{Sim}($ )
B- Não ( )

4- Tem dor na barriga frequente ou desconforto abdominal?
A- Sim ( )
B- Não ( )

Se tem dor abdominal onde é a dor?

C- No baixo ventre (abdômen inferior) ( ) D- No abdômen superior ( )

5- Sente alguma dor, coceira, ardência, ou elevação (tumoração) no ânus nos últimos meses?
A- Sim ( )
B- Não ( )

6- Perdeu o apetite nos últimos meses?

A- $\operatorname{Sim}($ )

B - Não ( )

7- Fez algum exame (hemograma) que deu Anemia?

A - $\operatorname{Sim}()$

B - Não ( )

8- Tem tido fraqueza (cansaço) constante nos últimos meses?

A - Sim ( )

B - Não ( )

9- Tem náuseas e/ou vômitos?

A - $\operatorname{Sim}()$

B - Não ( )

10- Você já fez exames como a colonoscopia?
A - Sim ( )
B - Não ( )

11-Você já teve pólipos intestinais?

A - Sim ( ) B Bão ( )

12- Em relação a seus familiares mais próximos: Pais, irmãos e avós:
Alguém teve câncer?
A- $\operatorname{Sim}($ )
B - Não ( )
Aonde?
C - Intestino ( )
D - Mama ( )
E - Estômago ( )
F - Ovários ( )
Quem teve?
G- Pais ( )
H-Avós ( )
I- Irmãos ( )
J-Tios ( )
13- Você:
A- É fumante ( )
B- Bebe regularmente ( )
C- Come gorduras e frituras sempre ( )
D- come verduras e frutas sempre ( )

14- Você já teve câncer?

A- $\operatorname{Sim}()$

B- Não ( )

Aonde?

C- Próstata ( )

D- Mama ( )

E- Estômago ( )

F- Ovários ( )

G- Útero ( ) 